\title{
Pratiques
}

Linguistique, littérature, didactique

\section{Recherche et différenciations socio-scolaires}

\section{Élisabeth Bautier}

\section{(2) OpenEdition \\ Journals}

Édition électronique

URL : http://journals.openedition.org/pratiques/1489

DOI : 10.4000/pratiques. 1489

ISSN : 2425-2042

\section{Éditeur}

Centre de recherche sur les médiations (CREM)

\section{Édition imprimée}

Date de publication : 15 juin 2010

Pagination : 17-21

\section{Référence électronique}

Élisabeth Bautier, "Recherche et différenciations socio-scolaires », Pratiques [En ligne], 145-146 | 2010, mis en ligne le 15 juin 2010, consulté le 01 mai 2019. URL : http:// journals.openedition.org/pratiques/1489; DOI : 10.4000/pratiques.1489 


\section{Recherche et différenciations socio-scolaires}

Si c'est la question de la légitimité des recherches en didactique qui est posée, mon propre point de vue ici en manque : n'ayant pas moi-même d'appartenance institutionnelle ou scientifique clairement didactique. En revanche, je peux (m')interroger sur la place des recherches en didactique (du français) quand on travaille, comme je tente de le faire, à comprendre les inégalités scolaires et sociales devant les apprentissages et le rôle que jouent les pratiques ordinaires de travail scolaire mises en place par les enseignants. Sur ce point force est de constater que nombre de recherches en didactique ne portent que peu sur ces questions ou sont ambiguës et, en tout état de cause, n'ont pas apporté les changements attendus par ceux qui, dans la deuxième moitié des années soixante avaient travaillé à lutter contre les inégalités sociales liées à la langue française à l'école et élaboré le Plan Rouchette.

\section{Légitimité scientifique et légitimité institutionnelle}

La distinction entre ces deux types de légitimité n'est pas vaine. Si la question de la légitimité scientifique des recherches ne se pose pas, en tout cas, pas davantage que dans d'autres domaines de recherche en SHS, elle est non dépourvue de sens du point de vue institutionnel, puisqu'il n'y a pas de section de CNU de didactique. Les docteurs en didactique du français se présentent ainsi à la qualification soit en sciences du langage, soit en sciences de l'éducation, soit en psychologie; il est vrai que les critères de légitimité scientifique des recherches variant en fonction des sections de $\mathrm{CNU}$, un certain flou au regard des critères de scientificité s'installe.

« Même » en sciences de l'éducation, les recherches en didactique ne sont pas $a$ priori valorisées, les questions scolaires, il est vrai, n'y occupent pas non plus une place prépondérante. Historiquement, les Sciences de l'éducation ont d'abord «accueilli » les didactiques des STAPS, puis peu à peu celles d'autres disciplines d'enseignement comme les langues secondes et étrangères et la langue maternelle, mais à certaines conditions. Les recherches en didactique qui s'inscrivent en sciences de l'éducation, ne peuvent se « réduire » à la présentation d'ingénierie didactique, l'accent se doit d'être mis sur la contextualisation des situations d'enseignement et d'apprentissage visant à prendre en considération la pluralité et l'hétérogénéité de leurs composantes à partir d'une pluralité de cadres théoriques conduisant à mobiliser simultanément des théories et savoirs sociologiques, cognitifs, ergonomiques, disciplinaires. On ne peut considérer un phénomène d'apprentissage et d'enseignement indépendamment de l'ensemble des éléments qui construisent la situation.

Mais au-delà de la question de la légitimité institutionnelle, une question reste posée, celle de la nature même des recherches, de leurs fondements, de leurs objectifs comme de leurs destinataires. Les recherches en didactique relèvent des sciences humaines et sociales et à ce titre, elles s'ancrent dans des questions sociales et humaines, à ce titre encore, elles se doivent d'identifier les questions auxquelles elles veulent répondre, elles se doivent aussi d'être autant fondamentales qu'appliquées ou finalisées. Là est peut-être une difficulté aujourd'hui : celle de reconnaître à quelles questions répondent nombre de recherche.

\section{De quelles recherches en didactique a-t-on besoin pour comprendre les difficultés des élèves ?}

Paradoxalement, pour la didactique du français, la place de la langue et du langage dans la question des inégalités sociales d'apprentissage est au cœur des questions 
d'enseignement, mais les réponses apportées par les recherches en didactique au cours des trente dernières années n'ont guère changé les situations, voire les ont aggravées. Il serait non seulement intéressant pour l'historien de la didactique d'en comprendre les raisons, mais également nécessaire d'y trouver les possibilités de changement d'orientation $^{(1)}$.

On peut tenter quelques hypothèses. Si la prise en compte du triangle didactique est une évidence, les recherches mettent souvent l'accent sur un des sommets du triangle plutôt que sur les relations entre les trois. Il en est ainsi de l'importance donnée à l'objet de savoir et aux modalités de son enseignement, en particulier en didactique du français ${ }^{(2)}$ qui a privilégié la recherche des objets enseignables. Ainsi, pour Yves Reuter (2007), les didactiques sont des « disciplines de recherche qui analysent les contenus (savoirs, savoir-faire...) en tant qu'ils sont objets d'enseignements et d'apprentissages référés/référables à des matières scolaires » Il ajoute que la spécificité des didactiques réside dans leur centration sur les contenus et sur leur interaction avec l'enseignement et les apprentissages. Il y a là sans doute une des premières causes : la didactique du français s'est beaucoup centré sur de « nouveaux objets » largement issus des recherches en linguistique. Le passage d'une grammaire de la langue à une linguistique des discours, des genres, de l'énonciation, en sont des exemples et non des moindres. Mais l'une des conséquences n'a-t-elle pas été le brouillage de la question principale : 1'apprentissage et l'acquisition par tous les élèves d'une langue et de ses usages qui permettent d'apprendre et d'écrire, d'élaborer et non " seulement » de produire des types de textes et des positions énonciatives. Cette remarque ne porte pas uniquement sur les situations d'écrit, la situation de l'oral dans les classes, ou plutôt de son enseignement relève de cette même logique.

Au-delà de ce brouillage, on peut encore parler de paradoxe quand, par le biais de la notion de discours et d'énonciation, on a pensé introduire le monde social et les élèves comme sujets sociaux; mais il s'est agi, certes de façon le plus souvent non volontaire et ignorée, de masquer justement le caractère profondément social de la langue, des discours et de ceux qui les produisent, c'est-à-dire inscrit dans des rapports sociaux, empêchant ce faisant, de prendre en considération les habitudes et connaissances des élèves dans leurs spécificités et modes de socialisation. Les acquisitions socialement différenciées des élèves n'étant guère prises en considération dans leur logique propre, les apprentissages nécessaires ou possibles ne le sont pas davantage. Si les objets d'enseignement n'ont cessé d'évoluer - et de devenir de plus en plus complexes -, la centration sur les modalités d'enseignement de ces objets a évacué peu à peu les évidences sociales qu'ils supposent. La langue et ses usages sont devenus « transparents ", pensés comme tels, dans le quotidien de la classe, alors même qu'ils sont de plus en plus opaques à une partie de plus en plus grande des élèves.

Dans cette même perspective, on peut encore considérer comme un paradoxe l'importance prise par les interactions et leurs analyses comme objets didactiques et d'enseignement. Deux raisons à cette évolution, l'importance de la communication comme nouvel objet d'apprentissage nécessaire, et l'affaiblissement du discours instructeur (B. Bernstein, 2007) au profit des échanges entre élèves et entre l'enseignant et les élèves, compte tenu des orientations « socioconstructivistes » actuellement dominantes. Même si cet affaiblissement a conduit nombre de recherches en didactique à travailler sur ces interactions, on y retrouve une conception de la langue, instrument de communication pensée comme transparente, d'accès partagé par tous.

(1) Les réflexions dans ce domaine sont alimentées par les échanges que je peux avoir avec Marceline Laparra.

(2) Ce n'était pas le cas dans certains courants de la didactique des mathématiques par exemple, que ce soit la théorie des situations (Brousseau) ou les travaux (D. Butlen et alii) qui ont analysé les pratiques enseignantes en ZEP. 
Tel n'est évidemment pas le cas, les enjeux dans ce domaine sont justement porteurs d'inégalités sociales. Et alors même que les relations entre oralité et écriture se trouvent au centre des échanges communicatifs lorsqu'ils portent sur de l'élaboration et des objets de savoirs, cette relation est restée et reste souvent impensée sur le plan didactique, encore moins comme étant à l'œuvre dans la construction des inégalités sociales à l'école. Sans doute faut-il noter ici que la psychologie (et l'analyse des opérations cognitives qu'elle permet) reste la discipline de référence des analyses didactiques, la sociologie et plus précisément la sociologie du langage et des apprentissages ayant donc toujours été de peu de ressources pour les chercheurs dans ce domaine.

\section{Recherches didactiques et dimension sociale des apprentissages langagiers}

Il ne s'agit évidemment pas ici de penser une homogénéité des positions épistémologiques et des objets, surtout en didactique du français! C. Garcia-Debanc (2006) a un point de vue assez différent quand elle définit « les travaux en didactique (comme) s'attachant à décrire et analyser les effets d'interventions d'enseignement ou de dispositifs de travail sur les apprentissages langagiers des élèves ».

Mais rares ont été jusqu'à présent les recherches en didactique du français qui ont pris en compte ces dimensions contextuelles et sociales qui convoquent de l'extériorité de la classe et même de la situation scolaire étudiée. Malgré les travaux de JeanFrançois Halté et ceux de Marceline Laparra qui très tôt se sont orientés vers une didactique centrée sur la réalité sociale des objets enseignés, sur leur histoire et les ambiguïtés auxquelles ils confrontent les élèves, cette orientation des recherches en didactique est longtemps restée marginale par rapport aux courants dominants et a eu peu d'impact sur la formation et les pratiques ordinaires de recherche.

Pourtant, qu'il s'agisse de recherches fondamentales ou de recherches visant une modification par la formation de manières de faire enseignantes dans un sens d'une plus grande réussite des apprentissages des élèves, les savoirs nécessaires issus de la didactique ne peuvent se construire que dans la prise en considération de l'interaction des différentes composantes des apprentissages dans les pratiques ordinaires de classe, composantes dont on ne peut exclure la dimension et la construction sociales. Il s'agit donc, d'une part, de (re)introduire cette inscription sociale des objets langagiers et linguistiques scolaires et, d'autre part, de penser les recherches de façon à ce qu'elles puissent la mettre en évidence.

L'évolution actuelle des recherches va certes dans ce sens puisque les corpus analysés le sont en situation réelle et dépassent largement la construction d'ingénierie didactique centrée sur l'apprentissage des discours et de leurs composantes pour prendre en considération des éléments hétérogènes. Mais en ce cas, outre l'interrogation sur la conception du langage qui sous-tend les analyses des situations, comme les situations elles-mêmes étudiées, il est nécessaire de s'interroger sur les conditions qui permettent de passer de recherches «étude de cas », ou d'observations fines mais ponctuelles, à des recherches qui permettent la construction de connaissances cumulables, comparables, qui autorisent un minimum de généricité quant aux résultats obtenus sur des problématiques et questions partagées. Parmi ces conditions, celle de la dimension temporelle dans lesquelles s'inscrivent les recherches apparaît importante, il ne peut s'agir que d'une temporalité longue, d'études longitudinales pour identifier les difficultés des élèves liées aux dispositifs mis en œuvre, aux manières de faire récurrentes et qui font cumul de difficultés et de non apprentissages, et les distinguer de celles qui relèvent de l'inscription normale dans le temps du développement et de l'apprentissage. Cette distinction entre difficultés liées au développement et à la progressivité de l'apprentissage (inscription temporelle qui fait sou- 
vent peu l'objet d'attention de la part de l'école) et celles liées au contexte et à la forme scolaire de l'apprentissage est importante (ce qui ne veut pas dire qu'elles ne se cumulent pas!) en cette période où les apprentissages de haut degré de littéracie et d'élaboration cognitive sont proposés aux élèves de plus en plus tôt et sans réel souci de progression. ${ }^{(3)}$

Une autre dimension temporelle vient croiser la précédente et s'inscrit davantage dans le travail enseignant, il s'agit de la gestion du temps didactique au-delà de celle du moment de la classe étudiée. Comme le soulignent N. Monnier et C. Amade Escot (2009), nombre d'enseignants gèrent une temporalité qui dépasse le moment d'enseignement et ce n'est qu'à la condition de prendre en considération cette dimension que ce qui se produit est intelligible au chercheur.

Travailler longitudinalement sur la temporalité des apprentissages et de 1'enseignement apparaît donc comme relevant d'une double nécessité : celle de rendre visible ce qui est réellement non appris, non acquis dans la durée et ce qui est récurrent dans la pratique enseignante et qui forme les façons à partir desquelles élèves et enseignant co-construisent la difficulté et ces «non- apprentissages ».

\section{Construire et étudier comment les élèves se saisissent des dispositifs}

La temporalité n'est évidemment pas la seule « entrée » dans ce travail d'intelligibilité de la difficulté, l'analyse des composantes des dispositifs et supports proposés au travail des élèves apparaît aujourd'hui d'importance compte tenu des modalités contemporaines d'enseignement. Le dispositif peut être défini comme une organisation spécifique matérielle, dans le temps et l'espace, des activités des élèves, réalisée à l'aide de supports et d'objets divers scolaires et moins scolaires, écrits, iconiques, collectifs et individuels, censée engager l'élève dans la réflexion et l'apprentissage.

L'affaiblissement depuis plusieurs années d'une parole enseignante instructive au profit de dispositifs « apprenants », souvent sophistiqués, devrait également avoir des conséquences sur les recherches en didactique et particulièrement en didactique de la langue maternelle. La sophistication des dispositifs actuels, la complexification des situations de production, leur inscription fréquente dans des évidences irréalistes de littéracie étendue partagée, comme dans des processus cognitifs de haut niveau de complexité méritent attention. Il apparaît en effet dans les recherches effectuées ou en cours sur ces questions ${ }^{(4)}$ que nombre de dispositifs utilisent des écrits très hétérogènes, supposant la familiarité avec des codes symboliques multiples et un usage élaboré de l'écrit dès le plus jeune âge des élèves sans que les apprentissages concernant ces attendus soient effectués.

Les recherches en didactique pourraient non seulement accorder de l'importance à l'élaboration de ces dispositifs, mais aussi - surtout ? - à la façon dont leurs différentes caractéristiques produisent des effets différenciés sur les élèves au-delà de leur valeur didactique propre. Il s'agirait de prendre en considération la pluralité des éléments cognitifs, sociologiques, culturels, langagiers qui interviennent et interagissent dans la construction de la situation pour l'élève. L'analyse de ces dispositifs est d'autant plus intéressante qu'ils correspondent à des représentations des enseignants, représentations de la discipline comme des élèves, représentation de l'apprentissage aussi, ils correspondent également à des doxas évidemment peu interrogées.

(3) Les recherches conduites dans le cadre du réseau Reseida tentent de présenter ces caractéristiques.

(4) Le travail de recherche de M. Delaborde (2009) synthétisé dans sa thèse en est un bon exemple. Voir aussi Bautier (ed.) 2006, Joigneaux (2009). 


\section{En conclusion}

Si donc aujourd'hui, les recherches en didactique se centrent davantage sur les analyses de pratiques enseignantes réelles, sur celles des interactions verbales élèves-élèves ou élèves-enseignants ou sur les productions langagières écrites des élèves pour y analyser les traces d'apprentissage ou de difficultés, si on voit se développer une didactique ascendante plus que descendante, les recherches restent de peu d'effets sur les pratiques. S'agit-il d'un moment de la recherche qui permet de rendre intelligibles les difficultés des élèves, s'agit-il de recherches très ponctuelles et dont le statut « hésite » entre l'exemple heuristique et l'étude de cas, ce qui rend difficile la généralisation des résultats pour la formation? Ou ces recherches peu prescriptives et mettant au jour la complexité de la classe et le travail didactique souvent « empêché » sont-elles trop ambiguës et « désespérantes » pour les enseignants, pris dans des injonctions et des doxas alors contradictoires (N. Monnier, C. Amade Escot, 2009)?

Au vu des réflexions précédentes, sans doute est-il également temps d'envisager au sein des recherches en didactique des recherches sur le curriculum : non pas sur le comment l'enseigner, mais sur le pourquoi et à quelles conditions tel ou tel objet de savoir ou telle ou telle activité cognitive (puisque de plus en plus ces activités priment sur les savoirs disciplinaires) peut-il (elle) et doit-il (elle) faire partie des apprentissages nécessaires et dans quelle progression ces derniers peuvent-ils se situer.

Élisabeth Bautier,

Paris 8, Circeft-Escol

\section{Références bibliographiques}

BAUTIER, É., (2006) (ed.) : Apprendre à l'école, apprendre l'école. Des risques de construction d'inégalités dès la maternelle. Lyon, Chronique sociale.

— (2009) : "Quand le discours pédagogique gêne la construction des usages littéraciés du langage », Pratiques « Ecrits de savoirs ", n 143-144.

Bernstein, B., (2007) : Pédagogie, contrôle symbolique et identité, Laval, Presses de l'université Laval.

Butlen, D., Masselot, P., PÉzard, M., (2004) : in Peltier M-L. (Ed) Dur, dur, dur d'enseigner en ZEP, Grenoble, La Pensée Sauvage.

DELABORDE, M., (2009) : Forme et sens de l'univers graphique en maternelle. Etudes de cas et enjeux didactiques, thèse de doctorat, Université de Metz.

GARCIA-DEBANC, C., (2006) : Intervention à l'école « Acquisition du langage et corpus en linguistique et psychologie », Moissac.

Joigneaux, C., (2009) : Des processus de différenciation dès l'école maternelle. Historicités plurielles et inégalité scolaire, Thèse de doctorat, université de Paris 8.

Monnier, N., AmADE-Escot, C., (2009) : «L'activité didactique empêchée : outil d'intelligibilité de la pratique enseignante en milieu difficile », Revue Française de Pédagogie, $\mathrm{n}^{\circ} 168$.

REUTER, Y., COHEN-AZRIA, C., (2007) : Dictionnaire des concepts fondamentaux des didactiques, Louvain la Neuve, De Boeck. 